\title{
Introducción: islas (des)imaginadas: de la matriz moderno-colonial al Caribe archipelágico ${ }^{1}$
}

La presente colección de artículos articula indagaciones en torno a las representaciones de las islas en textos literarios, objetos culturales, disciplinas académicas y diversos discursos de Occidente, desde múltiples geografías, cronologías y textualidades, en el cuestionamiento de un repositorio de lo insular intrínsecamente asociado con la expansión colonizadora de Europa hacia el resto del mundo. Estas lecturas interrogan múltiples zonas de ese repositorio desde una particular puesta en escena en el ámbito de lo que se denomina conflictivamente como "el Caribe", considerando tanto islas, fronteras continentales, como desarrollos transgeográficos de esa noción. De este modo, hacen emerger y revisitan imaginarios insulares constitutivos no sólo por los aparatos ideológicos de las invasiones imperiales euro-estadounidenses, sino también de consecutivas narrativas de identidad asociadas con la configuración de islas y "cuasi-islas" como Estados nacionales en el Caribe. Este número monográfico explora, entonces, múltiples genealogías simbólicas e intelectuales asociadas con las representaciones de las islas en distintos momentos del desarrollo literario, cultural, histórico, político e ideológico de dicha "geografía", proponiendo puntos de fuga hacia futuros posibles de lo insular/archipelágico en el Caribe y sus ámbitos globales de interacción. ${ }^{2}$

\section{Pensar lo insular desde la matriz moderno-colonial ${ }^{3}$}

En esta primera sección expongo algunas de las principales configuraciones teórico-críticas recientes acerca de las representaciones de las islas en el imaginario (post)occidental, en torno a las que gravitan los trabajos aquí reunidos. Con ese fin realizaré un acercamiento a los entrecruzamientos entre geografía y colonialidad, con el propósito de insistir en la necesidad de desnaturalizar el discurso geográfico y en la deconstrucción de las representaciones de las islas en el discurso occidental. El estudio acerca de las representaciones de las islas ha transitado por múltiples momentos teórico-críticos durante el siglo XX y lo que va del actual. Entre estos cabe mencionar: el análisis simbólico literario (Auden, 1950), la perspectiva psicoanalítica (Bachelard, 1942 y 1957), el enfoque mítico-simbólico de la antropología cultural (Durand, 1981), la reconfiguración de la geografía como disciplina (Lewis y Wigen, 1997; Dodds y Royle, 2003; Gillis, 2004, 2007) y la crítica postcolonial (Balasopoulos, 2008), sin intentar ser exhaustivo en esta enumeración.

\footnotetext{
${ }^{1}$ Introduction: 'De-Imagined' Islands: From the Modern/Colonial Matrix to Archipelagic Caribbean

${ }^{2}$ Agradezco a Silvia Valero, editora de Visitas al Patio, revista académica publicada por el programa de Lingüística y Literatura, de la Universidad de Cartagena, por su invitación a editar este número monográfico. También destaco la contribución de los autores de los artículos incluidos en esta colección, quienes demostraron gran compromiso durante todo el desarrollo de este proyecto editorial. Expreso por igual mi gratitud hacia los colegas que actuaron como pares evaluadores anónimos de los artículos remitidos a esta convocatoria.

${ }^{3}$ Presenté apartes de este texto en la ponencia titulada "Pensar como islas, actuar como archipiélago: de la matriz moderno colonial a los nacionalismos caribeños". XI Seminario Internacional de Estudios Caribeños. Cartagena de Indias: Universidad de Cartagena, Instituto Internacional de Estudios Caribeños, jul. 29-ago. 2, 2013. Agradezco a The University of Kentucky Graduate School y al Instituto Internacional de Estudios Caribeños por financiar mi participación en ese evento.
} 
En las últimas décadas, precisamente desde posiciones cercanas a la nueva geografía y la crítica poscolonial, se ha venido configurando el campo transdisciplinar de los estudios insulares (island studies), con mayor visibilidad en contextos lingüísticos anglófonos (Moles, 1982; Depraetere, 1990-1991, 2008; McCall, 1994 y 1996; Baldacchino, 2005, 2006; Hay, 2006; Balasopoulos, 2008). Los estudios insulares se constituyen en una perspectiva teórica-metodológica hacia el fenómeno de las islas que resulta de búsquedas por rebasar aproximaciones previas como los estudios simbólicos y la geografía moderna. En el caso de los estudios simbólicos sobre las islas, los estudios insulares reaccionan ante su matriz esencialista que desatiende los contextos de producción y reproducción mítico-simbólica. Por otro lado, se constituyen también en síntoma de un rechazo de la supuesta condición geográfico-natural de las islas, las que han pasado a ser comprendidas, como respuesta, como parte de representaciones culturales y discursos sobre la "naturaleza".

Estas nuevas configuraciones teóricas obedecerían a la emergencia de un creciente interés, desde las ciencias sociales y las humanidades contemporáneas, en apelar a la condición material y metafórica de las islas, es decir, a sus atributivos físicos y literarios, y a las repercusiones de estos atributos en la conformación de nacionalismos territoriales (Dodds y Royle, 2003: 487). ${ }^{4}$ En contraposición con este cuestionamiento de la noción de "isla", se plantea la emergencia de las islas oceánicas como una relevante metáfora social, articulada a la expansión colonial a través de los océanos a partir del siglo XVI (Groove, 1995: 24) y que continúa desarrollándose, entre otros, según lo han señalado Dodds y Royle, en el campo de las narrativas de la identidad cultural (2003: 489). Estos desplazamientos teóricos apuntan a reconocer que el lugar ocupado por las islas en el imaginario de Occidente tiene que ver más con condicionamientos históricos y culturales que con unos dispositivos físicos que puedan diferenciarlas de otros espacios geográficos. ${ }^{5}$ De ese modo, como ha indicado John R. Gillis, la percepción europea de las islas no es sólo consecuencia de las condiciones físicas de estas, sino igualmente un resultado de la "cultura continental occidental" (2007: 274).

Un desdoblamiento de esa matriz moderno-colonial se identifica con el papel crucial de las representaciones de las islas como el Otro en la constitución de determinadas formaciones nacionalistas. Es importante considerar, como ya he anunciado, la existencia de posturas que sostienen que la propia realidad geográfica insular conduciría a esa distinción y separación nacional:

National states hang together both by identifying external Others against whom they compare and contrast themselves and by making use of internal differences that symbolize the coercive and cultural challenges facing state formation. Boundary islands seem to offer specific advantages with respect to both elements of political boundary definition and maintenance. They are physically and often culturally distinctive but they are close by and within the official national territory... (Agnew 309) ${ }^{6}$

\footnotetext{
${ }^{4}$ En relación con la deconstrucción del carácter físico de las islas véase Dening, 1980 y Carter, 1999.

${ }^{5} \mathrm{Al}$ respecto, Gillis insiste en que los geógrafos han comenzado a cuestionarse las diferencias categoriales atribuidas a las islas y los continentes, lo que haría parte de un esfuerzo por abordar nociones como las de islas y continentes, así como las de océanos, playas, cañones, bosques y montañas, como construcciones culturales, producto más de la historia que de la naturaleza (2007: 277). Véase también: Corbin, 1994; Lewis y Wigen, 1997; Steinberg, 2001.

${ }^{6}$ Véase también Peckham, 2003.
} 
Este planteamiento de J. Agnew, enunciado en su análisis del caso de la isla de Sicilia como parte del territorio nacional italiano, contribuye a pensar en la situación de otras islas que, como buena parte de las caribeñas, constituyen -en sí mismas- estados nacionales. En este sentido, como proponen también Dodds \& Royle, lejos de ser periféricas en la comprensión del nacionalismo y el Estado-Nación moderno, las narrativas insulares cumplen un importante papel en el estudio de la formación de imaginarios nacionales (495). En esa misma dirección, cabe destacar un giro en el contexto caribeño hacia el estudio de la emergencia del pensamiento colonial resultado de la dominación europea. Esto implica la confrontación con proyectos de élite involucrados en la construcción de imaginarios de naciones insulares y regiones "cuasinsulares". Estos abordajes apuntan a desarticular dispositivos de memorias nacionales excluyentes que hacen de la exaltación del paisaje insular, como símbolo de una identidad nacional idealizada, un estereotipo de negación del carácter histórico de la explotación esclavista. Dichas construcciones, elaboradas principalmente durante el siglo XIX y retomadas por naciones independientes y/o neocoloniales durante la primera mitad del siglo XX, tuvieron como objetivo sublimar el papel de la explotación esclavista en el Caribe, a la vez que negar el aporte de las comunidades indígenas y africanas esclavizadas a la formación de sus culturales nacionales. Estas estrategias identitarias compartían sospechosamente la exaltación del paisaje insular como símbolo de una identidad nacional idealizada característica de islas pequeñas. A través de la presentación de la belleza de las islas como modelos idílicos, y muchas veces turísticos, se suprimía la memoria de la crueldad de la plantación esclavista como modelo económico y la complicidad de los europeos y criollos que participaron de ella. Luego de haber presentado someramente algunos de los desarrollos de los estudios insulares en su estado actual, en la próxima sección reseñaré la aparición del enfoque denominado como "estudios archipelágicos", señalando algunas de sus implicaciones teóricas para el orden actual de producción de conocimientos sobre las islas.

\section{El lado archipelágico de los estudios insulares anglófonos}

Hacia principios de los '80 comenzó a configurarse en el ámbito de los estudios caribeños un "pensamiento archipelágico" (archipelagic thinking) (Walcott, 1974; Brathwaite, 1983; Benítez-Rojo, 1989; Glissant, 1990), que impactó en la conformación de una subárea de los estudios insulares anglófonos o quizás de nuevo campo de estudios denominado actualmente como "estudios archipelágicos" (archipelagic studies) (DeLoughrey, 2001, 2007; Stratford, et al., 2011; Pugh, 2013, 2018; Murray, 2018). Los estudios archipelágicos responsabilizan a líneas de investigación previas de los estudios insulares de producir un "discurso dominante acerca y sobre las islas y los isleños en vez de con, desde y para ellos (Stratford, et al., 2011: 114). La principal disidencia de lo archipelágico radicaría entonces en su propuesta de superación de la perspectiva de estudio de las islas como sólo una relación tierra/mar e isla/continente, para transitar hacia una dimensión epistemológica que aborde los "espacios insulares como interrelacionados, constituidos mutuamente y co-construidos" (113). Algunas de las líneas de investigación más recientes asumen a "Envisioning the Archipelago" (Stratford, et al., 2011) como manifiesto constitutivo de los estudios

${ }^{7}$ Las traducciones son mías, a no ser que se indique otra cosa. 
archipelágicos y los identifican como un resultado del "giro espacial" de las ciencias sociales y las humanidades (Pugh, 2013: 12-14), llegando a proponer el advenimiento de un "giro archipelágico" (Archipelagic turn) en sí (Murray, 2018).

En esa dirección, Jonathan Pugh avanza en lo que considero una intensión de desnaturalizar y desterritorializar la propuesta de "pensar con el archipiélago" (thinking with the archipelago) (2013: 10-12), en la que cabe identificar dos momentos de expansión y aceleración. El primero consiste en la incorporación al objeto insular/archipelágico de "archipiélagos manufacturados", en referencia a conjuntos de turbinas de viento, bases militares insulares e "islas móviles" (portaviones, buques petroleros, etc.) (12). El segundo y más conflictivo momento de este proyecto apunta a separar las islas y/o archipiélagos de un "contenedor territorial predefinido" (predefined territorial container) (13), es decir, de su especificidad territorial en cuestión, para transar a "thinking with the archipelago" como una categoría o bien común a ser implementada en el ámbito general de los estudios sobre la cultura (14). No sorprende que Pugh base lo más sustantivo de su proposición en el análisis de amplias zonas de la teoría caribeña o "pensamiento caribeño". Es decir, que intente estudiar lo insular/archipelágico -"thinking with the archipelago"- desde una espacio-temporalidad euro-norteamericana, no-marcada, desterritorializada, postcolonial, teórica, en fin, globalmente exportable. Esta operación de expropiación y domesticación epistemológica no le es posible sin basarse mayormente en el estudio de autores caribeños -principalmente anglófonos y francófonoscomo: Derek Walcott, Wilson Harris, Kamau Brathwaite, Edouard Glissant y Antonio Benítez Rojo, borrando de paso la materialidad y especificidad de eso que llamamos islas y archipiélagos.

A esta altura de la discusión, no cabe duda de la culpa metafisica que cargan los estudios insulares/archipelágicos, como resultado de la limitación sistémica propia del actual orden global de producción y circulación de conocimientos. Me refiero, primero, a que siguen siendo modernamente anglófonos. A que, de igual modo, reinciden en la producción de un discurso [severamente] dominante sobre las islas, generado en grandes centros académicos de Australia, Canadá, Estados Unidos e Inglaterra. Y, finalmente, lo que considero más grave, continúan el legado de asimilar, expropiar y re-exportar saberes teóricos producidos en otras zonas del mundo, reforzando de ese modo la tradición impuesta por la modernidad colonial de que el Otro marcado que es el "Sur global" no está autorizado para producir Teoría (con mayúsculas). El objeto no alcanzado de los "archipelagic/island studies" continúa siendo, entonces, la deseada (des)imaginación de las representaciones de islas Otras hasta ahora recicladas por el saber medieval, (re)producidas por los discursos de expansión imperial, y reinstaladas y re-exportadas por una academia euro-norteamericana continentalizada, legado ella misma de esas estructuras moderno-coloniales.

\section{Hacia unos estudios insulares/archipelágicos críticos desde el Caribe}

A continuación esbozo el estado del arte de un campo de los estudios insulares/archipelágicos críticos, en el que identifico momentos de desplazamiento, disidencia y reformulación de los estudios insulares/archipelágicos hegemónicos, a través de la interrelación de proyectos de (des) imaginación de las islas en el Caribe, enunciados mayormente desde glocalidades diaspóricas de 
la academia euro-norteamericana. ${ }^{8}$ La isla que se repite: el Caribe y la perspectiva posmoderna (1989), de Antonio Benítez Rojo, constituye un texto de carácter fundacional en este campo, que desmonta oposiciones no excluyentes entre una máquina occidental (teorética/epistemológica/de poder) y una caribeña (poética/teleológica/de resistencia), para identificar repeticiones no superpuestas de condiciones y lecturas que lo conducen a la noción de un meta-archipiélago caribeño, a la vez metageográfico y metaepistemológico.

Casi veinte años después, en Out of Bounds: Islands and the Demarcation of Identity in the Hispanic Caribbean (2008), Dara E. Goldman realiza importantes aportes al estudio de la desesencialización y desterritorialización de las representaciones insulares, al identificar -por medio del análisis del desplazamiento, proliferación, diasporización y globalización del tropo de la isla como nación- la configuración de un modo retórico donde se renegociarían relaciones sociales de poder entre el adentro y el afuera de la islas. Cabe resaltar también en este escenario la contribución de Francisco-J. Hernández Adrián a la configuración de unos estudios insulares transatlánticos que conecten el análisis de literatura, cine, artes plásticas y otros discursos archipelágicos del Caribe, las Canarias y de un Sur global, como campo para la coordinación de estrategias críticas y políticas contra la reinscripción de las totalidades eurocéntricas (Hernández Adrián, 2006; Metzger, Hernández Adrián y Crichlow, 2014, etc.).

Destacan también por su participación en la reformulación de unos estudios insulares/archipelágicos críticos, autores como Carmen Cañete Quesada, Beatriz Llenín-Figueroa, Yolanda MartínezSan Miguel y Kevin Sedeño-Guillén, todos presentes en esta colección de artículos. Con su libro El exilio español ante los programas de identidad cultural en el Caribe insular (1934-1956) (2011), Cañete Quesada propone una interrogación transatlántica de la representación de las islas en notables proyectos identitarios de Puerto Rico, Cuba y República Dominicana que sistematiza la viabilidad de una mirada postnacional y exílica de dichas representaciones, visibilizando interrelaciones entre textualidades españolas exílicas y caribeñas, soslayadas desde la perspectiva de las literaturas nacionales. Por su parte, Llenín-Figueroa (2012, 2014a, 2014b, 2016), desde lo teórico-crítico, lo creativo y el activismo, participa de una configuración crítica de lo insular/ archipelágico que interpela lo colonial patriarcal desde lo político y lo comunitario. Su proyecto de libro en progreso Archipiélago entre lo atroz y lo luminoso: hacia los futuros decoloniales de Puerto Rico (título preliminar), explora las interrelaciones entre confederaciones caribeñas, relaciones archipelágicas, subjetividad antillana e historia marítima en proyectos políticos y artísticos puertorriqueños.

De igual modo, Martínez-San Miguel (2017, 2018, 2019, 2020) ha vuelto central en sus investigaciones el estudio de las articulaciones de lo colonial y lo poscolonial en la configuración de redes archipelágicas caribeñas, llamando la atención acerca de conexiones entre textualidades y políticas de lo insular, y dialogando críticamente con los estudios insulares/archipelágicos hegemónicos.

${ }^{8}$ No considero aquí textos realmente significativos para el pensamiento y la teoría insular del siglo XX como Antonio S. Pedreira, Insularismo (1934); José Lezama Lima, "Coloquio con Juan Ramón Jiménez" (1938); Virgilio Piñera, "La isla en peso" (1943), entre otros, previos a esta cronología de la institucionalización académica de los estudios sobre las islas. 
Esta por aparecer el volumen que ha coeditado bajo el título Contemporary Archipelagic Thinking: Towards New Comparative Methodologies and Disciplinary Formations (2020). Por último, SedeñoGuillén $(2011,2012,2015,2019)$ ha publicado ampliamente acerca de representaciones de las islas en la poesía cubana y colombiana, exiliados republicanos españoles y construcción de imaginarios insulares caribeños y acerca de una genealogía/arqueología de lo insular en el imaginario occidental. Su principal ámbito de investigación gira en torno al estudio de la insularidad caribeña como parte de una matriz global de representaciones moderno-coloniales de las islas. Este recorrido bibliográfico, y las propias propuestas incluidas en este número monográfico -que son reseñadas en la última sección de esta introducción- indican la vitalidad y visibilidad que han empezado a tener en español los estudios insulares/archipelágicos, en conexión con campos conexos como estudios caribeños, estudios atlánticos, estudios transatlánticos y estudios coloniales, entre otros.

\section{Un corpus global de textualidades insulares/archipelágicas}

Las propuestas incluidas en "Islas (des)imaginadas: de la matriz moderno-colonial al Caribe archipelágico" abordan una extensa e intrincada red de textos, artefactos, acontecimientos y prácticas en que prima la interconexión: cartografía del Caribe de finales del siglo XVII en relación con la narrativa diaspórica de Tiphanie Yanique, de las Islas Vírgenes en los Estados Unidos del XXI (Martínez-San Miguel); conexiones de la poesía de las independencias cubana e irlandesa de fines del siglo XIX en José Martí y W. B. Yeats (Sedeño-Guillén); debates de representación de lo americano/caribeño en la ensayística cubana y española (exílica) de Eugenio Fernández Granell y Alejo Carpentier, en la primera mitad del siglo XX (Cañete Quesada); fugas de lo insular en la poesía cubana de Virgilio Piñera de los '70 del mismo siglo (Rosario Ortiz); narrativas de la isla de Lenito Robinson Bent y Juan Ramírez Dawkins — en español, inglés y kreole—, del archipiélago de San Andrés, Providencia y Santa Catalina (Colombia) de entre siglos (Builes Vélez y Pérez Peña); las islas como laboratorio de experimentación del capital colonial patriarcal, sus articulaciones con las experiencias archipelágicas del CARICOM y la West Indies Federation, y las prácticas de resistencia y soberanía comunitaria en Puerto Rico de meses recientes (Llenín Figueroa).

Esta colección inicia con una propuesta en la que Yolanda Martínez-San Miguel entrelaza la lectura de mapas y textos europeos acerca del Caribe de los siglos XVII y XVIII, con cuentos contemporáneos de las literaturas diásporas caribeñas en los Estados Unidos. Su artículo ilustra, primero, un procedimiento de análisis que identifica, por medio del análisis de la representación del "archipiélago mexicano" en el mapa "Isole Antili, La Cuba e la Spagnuola" (1689), la centralidad del archipiélago como categoría geopolítica de vinculación colonial de Europa y las Américas. En un segundo momento, con la interpretación del cuento "The Bridge Stores: A short Collection" (2010), de la escritora Tiphanie Yanique (Islas Vírgenes estadounidenses), Martínez-San Miguel identifica la repetición de legados del "archipiélago imperial/colonial" en imaginarios caribeños contemporáneos. Este estudio logra así documentar la capacidad explicativa de lo archipelágico en la reconexión de las experiencias de islas imaginadas como coloniales, proponiendo una vía de deconstrucción de lo colonial, imperial e imperialista euro-estadounidense. 
En el artículo siguiente, Kevin Sedeño-Guillén analiza comparativamente los poemas "Abdala" (1869), de José Martí y The Wanderings of Oisin (1889), de W. B. Yeats, desde un contexto transimperial, translingüístico y transatlántico de lo insular, que le permite evidenciar ciertos desplazamientos espaciales y temporales que signan ambos textos. En "Abdala", apunta el traslado del escenario de representación de la isla caribeña a una extinta nación africana. Mientras que, en Yeats, enfatiza la actualización de los orígenes míticos de la nación irlandesa. Sedeño-Guillén propone que estos desplazamientos constituyen estrategias comunes de resistencia y oposición a la vigilancia y control ejercidos desde las respectivas metrópolis coloniales, llamando la atención hacia las poderosas articulaciones existentes en los textos postcoloniales estudiados entre la condición insular y la situación colonial.

En similar dinámica de interrelación, Carmen Cañete Quesada cuestiona la posicionalidad de ciertas textualidades caribeñas y europeas mediante el estudio de mecanismos y conflictos de legitimación en la representación de América. Para ello recurre al análisis comparativo de dos textos de índole ensayística: Isla cofre mítico (1951), de Eugenio F. Granell, escritor y pintor español exiliado; y "Lo real maravilloso en América" (1948), del escritor cubano-francés Alejo Carpentier. El artículo contrasta el imaginario de lo insular caribeño elaborado por Granell con la tesis identitaria sobre América Latina antes propuesta por Carpentier. Cañete Quesada presenta una seductora perspectiva de triangulación entre los autores citados y las teorías surrealistas -en particular con el libro Martinique charmeuse de serpents (1948), de André Bretón- que revela la inestabilidad en los autores estudiados de sus posiciones de enunciación de lo insular y lo americano.

Situando su interés en las dos décadas después del período comprendido en el artículo previo, Félix Miguel Rosario Ortiz emprende una lectura de "La isla en peso" (1943), del cubano Virgilio Piñera, desde la perspectiva de las "narrativas de la persecución". En su análisis de este poema emblemático de la literatura cubana acerca de la insularidad, Rosario Ortiz revela la angustia y ensimismamiento propios del espacio de la isla en su condición de metacárcel, aprisionada a la vez en un contexto caótico y violento del agua del mar. A su vez, con un agudo análisis crítico, camuflado en la densidad de lenguajes poéticos y filosóficos, este estudio dialoga productivamente con la amplia tradición de lecturas del poema piñeriano. Como resultado, lo resitúa en un propósito de exorcización, a la vez poética y política, del "razonamiento paranoico" de Piñera como artista perseguido político, que privilegia su percepción negativa de la condición insular y su intento de escape del aparato ideológico de una fijeza espacial, evocando un momento de álgida confrontación entre el gesto archipelágico de Piñera y el insularismo lezamiano-origenista.

En la misma línea de indagación acerca de las literaturas insulares caribeñas de finales del siglo XX e inicios del XXI, Ana Elena Builes Vélez y Melissa Pérez Peña proponen un reencuentro con teorías del Caribe que localizan su propuesta en el ámbito transdisciplinar de los estudios caribeños. Dicha instancia teórica sustenta su propósito de abandonar una perspectiva de estudio desde la "poetización caribeña", desde la playa al estilo odisíaco, por una que se adentre en la materia literaria insular desde las propias islas, que le daría la palabra a Circe. Este adentramiento las 
conduce al análisis de una zona compleja de la narrativa de los escritores Lenito Robinson-Bent y Juan Ramírez Dawkins, de la isla de Providencia (Colombia), con lo que Builes Vélez y Pérez Peña ponen en juego profundos conflictos políticos, territoriales, lingüísticos, de lo cultural y lo literario en su sentido más amplio, consecuencia de las distintas colonizaciones vividas por ese archipiélago y su creciente "continentalización”.

Finalmente, Beatriz Llenín Figueroa defiende - a través de la contraposición de dos momentos en sí confrontados: la isla-laboratorio y el archipiélago-experimento - una visión política, actual y crucial de lo insular/archipelágico caribeño en el complejo contexto económico, político y social de las "islas" de Puerto Rico. El artículo documenta cómo Puerto Rico ha sido instrumentalizado como isla-laboratorio para la experimentación y puesta en funcionamiento de técnicas de dominación colonial y poscolonial, que explotan la insularidad a favor de la transformación y expansión del capital colonial patriarcal. Este primer momento crítico conduce a Llenín Figueroa a proponer las soberanías comunitarias como expresión de democracia participativa, por un lado, y la integración regional en forma de conexión archipelágica, como estrategias decoloniales para hacer de Puerto Rico un archipiélago-experimento de emancipación contra el poder imperial de los Estados Unidos.

La conexión y repetición -en el sentido de Benítez-Rojo- de una isla que se repite siempre de forma distinta, ya sea como salida decolonial de lo insular/archipelágico que alienta esta propuesta de Llenín Figueroa o en la insistencia compartida por Martínez-San Miguel -en su contribución inicial-, y por los demás colaboradores de este número monográfico, conduce a recalcar la necesidad de continuar interrogando las condiciones y circunstancias del "archipiélago imperial/colonial" caribeño, como punto de fuga de los legados de lo insular (pos)colonial.

\section{BIBLIOGRAFÍA}

Agnew, J. (2000). Italy's Island Other: Sicily’s History in the Modern Italian Body Politic. Emergences, 10, 301-311. Cit. en Dodds \& Royle, 2003: 492.

Auden, W. H. (1950/1967). The enchafèd Flood: Or, the Romantic Iconography of the Sea. New York: Vintage Books.

Bachelard, G. (1942/1996). El agua y los sueños: ensayo sobre la imaginación de la materia. 2 reimpres. Trad. Ida Vitale. México: Fondo de Cultura Económica.

Bachelard, G. (1957/1995). La poética del espacio. Trad. Ernestina de Champourcin. 2 ed. 2 reimpres. Bogotá: FCE.

Balasopoulos, A. (2008). Nesologies: Island Form and Postcolonial Geopoetics. Postcolonial Studies, 11(1), 9-26. 
Baldacchino, G. (2005). Islands - Objects of Representation. Geografiska Annaler, 87 B.4, 247251. Cit. en Hay, 2006: 19-20.

Baldacchino, G. (2006). "Island, Island Studies, Island Studies Journal". Island Studies Journal, 1(1), 3-18. Recuperado de https://www.islandstudies.ca/sites/vre2.upei.ca.islandstudies.ca/ files/u2/ISJ-1-1-2006-Baldacchino-pp3-18.pdf

Benítez-Rojo, A. (2010). La isla que se repite: el Caribe y la perspectiva posmoderna. 1989. Ed. Rita Molinero. San Juan, PR: Editorial Plaza Mayor.

Brathwaite, E. K. (1983). Caribbean Culture: Two Paradigms. En: J. Martini, ed. Missile and Capsule (pp. 9-54). Bremen, Universitat Bremen.

Cañete Quesada, C. (2011). El exilio español ante los programas de identidad cultural en el Caribe insular (1934-1956). Frankfurt/Madrid: Vervuert/Iberoamericana.

Carter, P. (1999). Dark with Excess of Bright: Mapping the Coastline of Knowledge. En: D. Cosgrove, ed. Mappings (pp. 125-168). London: Reaktion Books.

Corbin, A. (1994). The Lure of the Sea: The Discovery of the Seaside in the Western World, 17801840. Berkeley: University of California Press.

DeLoughrey, E. (2001). "The Litany of Islands, the Rosary of Archipelagoes": Caribbean \& Pacific Archipelagraphy. Ariel: Review of International English Literature, 32(1), 21-51.

DeLoughrey, E. (2007). Routes and Roots: Navigating Caribbean and Pacific Island Literatures. Honolulu HI: University of Hawai'i Press.

Dening, G. (1980). Islands and Beaches: Discourse on a Silent Land, Marquesas 1774-1880. Honolulu: Dorsey Press.

Depraetere, C. (1990-1991). Le phénomène insulaire á l'échelle du globe: tailles, hiérarchies et formes des îles océanes. L'Éspace Géographique, 2, 126-134.

Depraetere, C. (2008). The Challenge of Nissology: A Global Outlook on the World Archipelago. Part I. Scene Setting the World Archipelago. Island Studies Journal, 3(1), 3-16. Recuperado de https://www.islandstudies.ca/sites/vre2.upei.ca.islandstudies.ca/files/ISJ-3-1-2008-Depraetere1-FINAL_0.pdf

Dodds, K. y S. A. Royle (2003). The historical geography of islands. Introduction: rethinking islands. Journal of Historical Geography, 29(4), 487-498. 
Durand, G. (1981). Las estructuras antropológicas de lo imaginario: introducción a la arquetipología general. Trad. Mauro Armino. Madrid: Taurus.

Gillis, J. R. (2004). Islands of the Mind: How the Human Imagination Created the Atlantic World. New York: Palgrave Macmillan. Cit. en Gillis, 2007.

Gillis, J. R. (2007). Island Sojourns. Geographical Review, 97(2), 274-287. ProQuest Science Journals.

Glissant, Edouard (2017). Poética de la relación. 1990. Trad. Senda Inés Sferco y Ana Paula Penchaszadeh. Bernal: Universidad Nacional de Quilmes.

Goldman, Dara E. (2008). Out of Bounds: Islands and the Demarcation of Identity in the Hispanic Caribbean. Lewisburg: Bucknell University Press.

Grove, R. (1995). Green Imperialism: Colonial Expansion, Tropical Island Edens and the Origins of Environmentalism. 1600-1860. Cambridge: Cambridge University Press. Cit. en Dodds \& Royle, 2003: 488.

Hay, P. (2006). A Phenomenology of Islands. Island Studies Journal, 1(1), 19-42. Recuperado de https://www.islandstudies.ca/sites/vre2.upei.ca.islandstudies.ca/files/u2/ISJ-1-1-2006-Haypp19-42.pdf

Hernández Adrián, Francisco-J. (2006). Atlantic Nessologies: Image, Territory, Value. Issue 1 "Rethinking Spain From Across the Seas". Studies in $20^{\text {th }} \& 21^{\text {st }}$ Century Literature, 30, Article 3. https://doi.org/10.4148/2334-4415.1613

Lewis, M. W. y K. E. Wigen (1997). The Myth of Continents: A Critique of Metageography. Berkeley: University of California Press.

Llenín-Figueroa, C. B. (2012). Imagined Islands: A Caribbean Tidalectics. (Tesis de doctorado). Duke University, Durham, NC. Recuperado de: https://dukespace.lib.duke.edu/dspace/bitstream/handle/10161/5420/LlennFigueroa_duke_0066D_11264.pdf?sequence=1

Llenín-Figueroa, C. B. (2014a). "The Island Blazed": A Blinding Light and Tiepolo’s Hound. Journal of Latin American Cultural Studies, 23(2), 173-191.

Llenín-Figueroa, C. B. (2014b). "I believe in the future of 'small countries:'" Édouard Glissant's Small, Archipelagic Scale in Dialogue with Other Caribbean Writers. Discourse: Journal for Theoretical Studies in Media and Culture, 36(1), Winter, 87-111. 
Llenín-Figueroa, C. B. (2016). "Armar una literatura de conexiones" en nuestra "patria líquida": el contexto caribeño de la ensayística de Marta Aponte Alsina. Caribbean Studies, 44(1), $29-45$.

Martínez-San Miguel, Y. (2017). Colonial and Mexican Archipelagoes: Reimagining Colonial Caribbean Studies. En: Archipelagic American Studies (pp. 155-173). Brian Roberts, Michelle Stephens, eds. Durham, NC: Duke University Press.

Martínez-San Miguel, Y. (2018). Colonialismo y decolonialidad archipielágica en el Caribe. Tabula Rasa, 29, 37-64. https://doi.org/10.25058/20112742.n29.03

Martínez-San Miguel, Y. (2019). Entre con/federaciones: Nicolás Guillén, Luis Palés Matos y Aimé Césaire. 80grados, jun., 28. Recuperado de http://www.80grados.net/entre-con-federaciones/

Martínez-San Miguel and Michelle Stephens, eds. (2020). Contemporary Archipelagic Thinking: Towards New Comparative Methodologies and Disciplinary Formations. Lanham, MD. Rowman \& Littlefield.

McMahon, Elizabeth, et al. (2011). "Envisioning the Archipelago". Island Studies Journal, 6(2), 113-130. Recuperado de https://ro.uow.edu.au/cgi/viewcontent.cgi?article=6404\&contex$\mathrm{t}=$ scipapers

McCall, G. (1994). Nissology: A Proposal for Consideration. Journal of the Pacific Society, 17(2-3), 1-8.

McCall, G. (1996). Nissology: A Debate and Discourse from Below, 1996. Cit. en Hay, 2006: 19.

Metzger, Sean; Francisco-J. Hernández Adrián y Michaeline Crichlow (2014). Introduction: Islands, Images, Imaginaries. Special issue "Islands, Images, Imaginaries". Third Text, 28(45), 333-343.

Moles, A. (1982). Nissonologie, ou Science des Îles. L’Éspace Géographique, 4, 281-289.

Murray, P. J. (2018). The archipelagic turn: nationhood, nationalism and early modern studies, 1997-2017. The Seventeenth Century, 33(4), 485-495. https://doi.org/10.1080/026811 $7 \mathrm{X} .2018 .1484637$

Peckham, R. S. (2003). The Uncertain State of Islands: National Identity and the Discourse of Islands. Journal of Historical Geography, 29, 499-515. 
Pugh, J. (2013). Island Movements: Thinking with the Archipelago. Island Studies Journal, 8(1), 9-24. Recuperado de https://www.islandstudies.ca/sites/islandstudies.ca/files/ISJ-8-1-2013Pugh_0.pdf

Pugh, J. (2018). Relationality and Island Studies in the Anthropocene. Island Studies Journal, 13(2), 93-110. Recuperado de https://www.islandstudies.ca/sites/default/files/ISJPughRelationalityAnthropocene.pdf

Sedeño-Guillén, K. (2011). Viaje iniciático de María Zambrano a la isla secreta: pensamiento insular y vivencia caribeña del exilio en Cuba y Puerto Rico. En M. Cámara y L. Ortega Hurtado (Eds.). María Zambrano: palabras para el mundo (pp. 91-104).. Newark, DE: Juan de la Cuesta Hispanic Monographs.

Sedeño-Guillén, K. (2012). “Como a tierra propia, como a patria suya, patria americana, patria insular": reflexiones insulares de tres andaluces en Cuba: Federico García Lorca, Juan Ramón Jiménez y María Zambrano. En H. Buffery, F. Lough, E. Marcer, y A. Sánchez (Eds.). Spanish Republican Exile Geographies (pp. 57-66). Birmingham, UK: University of Birmingham.

Sedeño-Guillén, K. (2015). Disciplinamiento vs. reinvención del espacio americano: teorías de lo costero/insular en José Lezama Lima. Visitas al Patio 9, 49-69. Recuperado de https:// revistas.unicartagena.edu.co/index.php/visitasalpatio/article/view/1748/1570

Sedeño-Guillén, K. (2019). Las islas de noche. San Juan de la Cruz, Martí y Lezama: extrañeza y nocturnidad hacia un imaginario insular cubano. Cuadernos de Literatura del Caribe e Hispanoamérica 29. DOI: http://dx.doi.org/10.15648/c1.29.2019.4

Steinberg, P. E. (2001). The Social Construction of the Ocean. Cambridge, England: Cambridge University Press.

Stratford, E. (2011). Envisioning the Archipelago. Island Studies Journal, 6(2), 113-130. Recuperado de https://www.islandstudies.ca/sites/islandstudies.ca/files/ISJ-6-2-2011-Stratford-et-al. pdf

Walcott, D. (1974). The Caribbean: culture or mimicry?. Journal of Interamerican Studies and World Affairs, 16(1), 3-13.

Kevin Sedeño-Guillén ${ }^{9}$

(Editor invitado)

${ }_{9}^{9}$ Doctor en Estudios Hispánicos, University of Kentucky. Profesor asistente visitante, Department of Spanish and Portuguese, Colorado College. Profesor del Programa de Lingüística y Literatura, Universidad de Cartagena. Correo electrónico: ksedenog@unicartagena.edu.co 